\title{
Variações fenotípicas em subpopulações de Davilla elliptica A. St.-Hil. (Dilleniaceae) e Byrsonima intermedia A. Juss. (Malpighiaceae) em uma área de transição cerrado-vereda
}

\author{
Léo Correia da Rocha Filho ${ }^{1}$ e Cecília Lomônaco ${ }^{2,3}$
}

\author{
Recebido em 31/10/2005. Aceito em 10/04/2006
}

\begin{abstract}
RESUMO - (Variações fenotípicas em subpopoulações de Davilla elliptica A. St.-Hil. (Dilleniaceae) e Byrsonima intermedia A. Juss. (Malpighiaceae) em uma área de transição cerrado-vereda). Foram descritas variações morfológicas em duas espécies de plantas arbustivas do cerrado. Para D. elliptica, a biomassa dos frutos e a altura das plantas foram maiores na borda da vereda. Contrariamente, verificou-se que o comprimento e a largura das folhas foram maiores na área de cerrado. Não houve diferenças entre as áreas com relação ao número de frutos por planta e formato do limbo foliar. Subpopulações de B. intermedia foram diferenciadas pela altura dos indivíduos, biomassa dos frutos e índice foliar, todos apresentando maiores valores no cerrado. Não foram constatadas diferenças entre as áreas no número de frutos por planta, comprimento das inflorescências, largura e comprimento da folhas. D. elliptica mostrou maior sucesso reprodutivo na área de vereda, onde ocorria com maior densidade, enquanto que $B$. intermedia apresentou melhor desempenho no ambiente do cerrado, embora diferenças nas densidades de ocorrência não tenham sido verificadas. Em comum, as espécies apresentaram padrões assincrônicos nos estádios de floração, iniciada mais tardiamente nas áreas de cerrado. Os dados obtidos confirmam a importância da heterogeneidade ambiental como fonte geradora de variabilidade fenotípica em caracteres ligados à história de vida das plantas.
\end{abstract}

Palavras-chave: plasticidade fenotípica, vereda, cerrado

\begin{abstract}
Phenotypic variation in Davilla elliptica A. St.-Hil. (Dilleniaceae) and Byrsonima intermedia A. Juss. (Malpighiaceae) subpopulations in a savanna/“vereda" transitional area). Morphological variations of two shrub species of the Brazilian savanna were described. For D. elliptica, fruit biomass and plant height were significantly larger in the "vereda" area, although leaf width, leaf length and leaf shape did not differ between areas. Subpopulations of $B$. intermedia differed in fruit biomass, plant height and leaf area index, all larger in the savanna area. No significant differences were detected between areas for number of fruits per plant, length of leaves and inflorescences and leaf width. D. elliptica showed better reproductive success in the "vereda" area, where it has greater density, whereas B. intermedia demonstrated better performance in the savanna area, although no difference was found in density between areas. On the other hand, both species presented a similar pattern of asynchronism of the flowering period, which began later in the savanna area. The data confirm the importance of phenotypic plasticity as a source of variability in plant life-history characters.
\end{abstract}

Key words: phenotypic plasticity, savanna, palm swamp

\section{Introdução}

A variabilidade fenotípica total (Vf) de um caráter em uma população é resultante não somente da variabilidade genotípica $(\mathrm{Vg})$ de seus membros, mas também da influência exercida pelo meio ambiente na determinação dos caracteres fenotípicos (Va). Assim, a variância total de um grupo populacional representa a soma de seus componentes genéticos e ambientais de variação, de modo que $\mathrm{Vf}=\mathrm{Vg}+\mathrm{Va}$ (Falconer 1989). O componente ambiental de variação, também denominado plasticidade fenotípica, é descrito como sendo a habilidade de ajuste morfológico e/ou fisiológico ao ambiente, sem que mudanças genéticas sejam necessárias (Bradshaw 1965; Price et al. 2003). Uma vez que a seleção natural atua sobre os fenótipos, e não diretamente sobre os genótipos, conhecer os componentes ambientais de variação, (i.e. as respostas plásticas de determinada população sob influências ambientais), é fundamental para a compreensão de como a população se estrutura no tempo e no espaço. Respostas plásticas podem ser adaptativas e, em ambientes heterogêneos, onde a população está sujeita à seleção disruptiva, há potencialidades para a

\footnotetext{
1 Universidade Federal do Paraná, Departamento de Zoologia, C. Postal 19020, 81531-980 Curitiba, PR, Brasil

2 Universidade Federal de Uberlândia, Instituto de Biologia, C. Postal 593, 38400-902 Uberlândia, MG, Brasil

3 Autor para correspondência: lomonaco@ufu.br
} 
formação de subpopulações (Thompson 1991). Assim, mesmo havendo plena possibilidade de fluxo gênico entre as subpopulações, por estas estarem muito próximas, diferenças morfológicas são geradas e mantidas ao longo do tempo entre as distintas áreas, por indução do ambiente, mesmo não ocorrendo modificações genotípicas.

O cerrado, dada sua complexidade vegetacional (Mares 1986) e diversidade de fisionomias, incluindo o cerrado sensu stricto, cerradão, campo cerrado, campo sujo e campo limpo (Coutinho 1978), é um ambiente propício para o estudo de componentes ambientais de variação em populações de plantas. $\mathrm{O}$ aumento ou manutenção da variabilidade de um grupo populacional é uma ferramenta importante para sua preservação, por torná-lo mais apto a sobreviver e reproduzir quando submetido a fortes pressões seletivas. Deste modo, saber como as plantas nativas do cerrado respondem à heterogeneidade ambiental por meio de respostas plásticas poderia ser muito útil para que programas de manejo e conservação de reservas ecológicas possam ser elaborados com maior eficiência. Isto porque espera-se que áreas com maior heterogeneidade ambiental possuam maior expressividade na variabilidade fenotípica em decorrência de componentes ambientais de variação.

Diferenças fenotípicas caracterizando subpopulações adjacentes foram registradas para algumas espécies vegetais em distintos ambientes do cerrado: Cabralea canjerana subsp. polytricha (A.Juss.) T.D. Penn. (Meliaceae), Eugenia calycina Cambess. (Myrtaceae) Acosmium dasycarpum (Vogel) Yakovlev (Fabaceae), Byrsonima crassa Nied. (Malpighiaceae), Caryocar brasiliense Cambess (Caryocaraceae), Matayba guianensis Aubl. (Sapindaceae), Miconia fallax DC. (Melastomataceae), Qualea parviflora Mart. (Vochysiaceae) e Roupala montana Aubl. (Proteaceae) (Fuzeto \& Lomônaco 2000; Cardoso \& Lomônaco 2003; N.S. Reis, dados não publicados). Esses trabalhos demonstraram que a heterogeneidade ambiental está, de fato, associada a uma grande variabilidade nos caracteres somáticos e reprodutivos, que muitas vezes possuem caráter adaptativo, visto que aumentam a sobrevivência e/ou potencial reprodutivo (Donohue 2003). Outra questão apontada pelos estudos mencionados é que diferentes espécies podem apresentar respostas plásticas distintas a um mesmo fator ambiental. Compreende-se, em decorrência disso, que um determinado fator ambiental (ou conjunto de fatores) interage de modo bastante peculiar com as distintas espécies vegetais, gerando diferentes respostas plásticas e que as implicações ecológicas e evolutivas desse fenômeno podem ser bastante relevantes para se compreender a dinâmica de estruturação das comunidades vegetais. Entretanto, para que padrões de repostas plásticas possam ser reconhecidos e descritos, dentro de diferentes níveis taxonômicos, são necessários dados adicionais, incluindo o estudo de novas espécies.

Assim, com o objetivo de ampliar o banco de dados sobre este assunto, o presente estudo trata das variações fenotípicas em Davilla elliptica A. St.-Hil. (Dilleniaceae) e Byrsonima intermedia A.Juss. (Malpighiaceae) em uma área heterogênea que apresenta gradiente de transição cerrado-vereda, no município de Uberlândia, MG.

\section{Material e métodos}

Área de Estudo - O estudo foi desenvolvido na RPPN (Reserva Particular do Patrimônio Natural) do Clube Caça \& Pesca Itororó de Uberlândia, MG (18 $55^{\circ}$ 'S e $48^{\circ} 17^{\prime} \mathrm{O}$ ). A reserva apresenta áreas de cerrado (sensu lato), que se divide entre as fisionomias de campo sujo e cerrado sensu stricto, sendo ainda entrecortadas por uma vereda. Esta última constitui-se de vegetação predominantemente herbácea, que se estabelece em áreas de nascentes (Castro 1980; Appolinário \& Schiavini 2002). As veredas possuem solos hidromórficos, mal drenados e com espessa camada de matéria orgânica mal decomposta sobre uma camada acinzentada (gleizada), resultante de ambiente de oxirredução (Sano \& Almeida 1998). Nas áreas de cerrado sensu-stricto que circunvizinham a vereda, predominam os Latossolos Vermelho Distróficos, moderadamente ácidos (Embrapa 1982; Cardoso \& Lomônaco 2003). O ecótono de transição cerrado sensu-stricto/vereda pode ser caracterizado visualmente pela gradativa redução da cobertura vegetal arbóreo-arbustiva e sombreamento, maiores no ambiente do cerrado sensu-stricto $(20$ a $50 \%)$ do que na vereda (5 a 10\%) (Sano \& Almeida 1998). O clima da região caracteriza-se por duas estações bem definidas: uma seca e outra úmida, sendo comum a ocorrência de geadas durante o inverno. Os índices pluviométricos oscilam anualmente em torno de $1.550 \mathrm{~mm}$. A temperatura média anual é de $22{ }^{\circ} \mathrm{C}$ (Nimer \& Brandão 1989).

Espécies estudadas - D. elliptica é uma planta de porte arbustivo, intensamente ramificada, conhecida 
popularmente como "lixeirinha", "lixeira-rasteira" e "lixinha", por apresentar a face superior de suas folhas bastante áspera, conferindo-lhe um aspecto de lixa. Possui folhas inteiras, alternas, elípticas e pecioladas e é amplamente encontrada no bioma Cerrado, preferindo solos arenosos (Pott \& Pott 1994). Suas flores são amarelas e efêmeras (duram apenas meio dia), com antese diurna. Seus frutos são envoltos por sépalas grandes e persistentes (Joly 1987).

Byrsonima intermedia apresenta porte arbustivo, e é popularmente conhecida como "muricizeiro", "murici", "muruci", "murici-do-campo" e "baga-detucano" (Lorenzi \& Matos 2002), ocorrendo principalmente em solos arenosos (Panizza 1998; Lorenzi 2000). Apresenta folhas inteiras, de disposição oposta, com estípulas intrapeciolares. As flores são vistosas, amarelas, hermafroditas, zigomorfas e reunidas em racemos terminais. A antese é diurna e as flores duram em média $48 \mathrm{~h}$. Os frutos são globosos, amarelos quando maduros e portam apenas uma semente (Joly 1987).

Exemplares das plantas estudadas foram depositados como material testemunho no Herbarium Uberlandensis, sob os números 32.195 e 29.995, para $D$. elliptica e $B$. intermedia, respectivamente.

Caracteres morfológicos analisados - Indivíduos adultos de $D$. elliptica e $B$. intermedia foram aleatoriamente identificados nas áreas adjacentes de cerrado sensu stricto $(\mathrm{n}=22)$ e vereda $(\mathrm{n}=22)$. Esses indivíduos foram medidos quanto à altura, utilizandose fita métrica, e, em cada planta, foi contado o número de frutos. Cerca de 170 frutos de ambas as espécies, provenientes de diferentes indivíduos de cada área, foram pesados com o uso de balança analítica. Para ambas as espécies foram coletadas, em 20 plantas, escolhidas de forma aleatória, as cinco folhas inteiras mais próximas à base dos ramos. $\mathrm{O}$ comprimento $(\mathrm{C})$ de cada folha foi medido com o uso de paquímetro e, no ponto médio, aferiu-se a medida da largura (L). Para a caracterização do formato da folha, calculou-se um índice foliar (IF), utilizando-se a seguinte fórmula $\mathrm{IF}=\mathrm{L} \mathrm{C}^{-1} . \mathrm{Em}$ B. intermedia foi medido, com auxílio de paquímetro, o comprimento de 200 inflorescências, oriundas de 40 indivíduos sorteados aleatoriamente, sendo 100 delas provenientes de cada área (cinco inflorescências de cada planta selecionada).

Foi determinado se havia sincronização no período de floração entre as subpopulações nas duas espécies, acompanhando-se em ambas as áreas o processo de produção e desenvolvimento de estruturas reprodutivas.
Para registro e comparação, foi realizada a categorização dos estádios de floração durante o período da manhã, em um único dia. Em $D$. elliptica, as fenofases foram caracterizadas em botão, botão em pré-antese, flor aberta e flor senescente. Para $B$. intermedia, os estádios considerados foram: botão fase I (de cor verde, de 1,06 a 1,91 mm), botão fase II (de cor verde-amarelada, de 2,10 a 4,22 mm), botão fase III (de cor amarela e em pré-antese), flor aberta, flor senescente, fruto verde e fruto maduro (coloração amarela). Foram consideradas nessa análise 22 inflorescências em cada área, uma por planta, para ambas as espécies.

A densidade de ocorrência das plantas, nas duas áreas, foi estimada estabelecendo-se 20 parcelas de $10 \times 10 \mathrm{~m}$ em cada área. Nessas parcelas foram contados todos os indivíduos reprodutivos presentes. A densidade foi obtida a partir da média dos valores de densidade (expressos em indivíduos $\mathrm{m}^{-2}$ ) entre as parcelas de cada área.

Análises estatísticas - Para verificar se as distribuições dos dados eram normais, foi utilizado o teste de Kolmogorov-Smirnov. Uma vez constatada a não normalidade dos dados, testes não paramétricos foram utilizados. Assim, diferenças significativas das variáveis obtidas entre as áreas de estudo foram verificadas pelo teste de Mann-Whitney. Uma tabela de contingência foi construída para averiguar se havia sincronia de floração entre as áreas de coleta (Zar 1984).

\section{Resultados}

Para D. elliptica, diferenças significativas entre as áreas foram obtidas quanto à altura dos indivíduos, biomassa dos frutos, largura e comprimento das folhas (Tab. 1). Na região de borda de vereda, a altura das plantas $(\mathrm{U}=120 ; \mathrm{P}=0,004)$ e a biomassa dos frutos $(\mathrm{U}=1652 ; \mathrm{P}<0,001)$ foram maiores quando comparadas aos valores encontrados na área de cerrado sensu stricto. Já o comprimento e a largura das folhas foram maiores na área de cerrado do que na borda de vereda $(\mathrm{U}=6774,5 ; \mathrm{P}<0,001$ e $\mathrm{U}=6863$; $\mathrm{P}<0,001$, respectivamente). Não houve diferenças significativas entre as áreas estudadas com relação ao número de frutos por planta $(\mathrm{U}=26 ; \mathrm{P}=0,795) \mathrm{e}$ índice foliar $(\mathrm{U}=5143 ; \mathrm{P}=0,727)$. Além disso, foi constatada assincronia no período de floração entre as áreas estudadas $\left(\chi^{2}=14,5 ; \mathrm{P}<0,05\right)$, sendo que a região de borda de vereda apresentou estádios de floração antecipados em relação à área de cerrado 
Tabela 1. Caracterização fenotípica (média e desvio padrão - DP), densidade e comparações (feitas pelo teste de Mann Whitney) em Davilla elliptica (A. St.-Hill) nas áreas de cerrado sensu stricto e borda de vereda da Reserva Vegetal do Clube Caça \& Pesca Itororó de Uberlândia, MG. (Índice foliar calculado pela razão entre largura e comprimento da folha).

\begin{tabular}{|c|c|c|c|c|c|c|c|c|}
\hline \multirow[t]{2}{*}{ Caracteres analisados } & \multicolumn{4}{|c|}{ Cerrado sensu stricto } & \multicolumn{4}{|c|}{ Borda de Vereda } \\
\hline & $\mathrm{N}$ & Média & $\mathrm{DP}$ & $\mathrm{N}$ & Média & DP & $\mathrm{U}$ & $\mathrm{P}$ \\
\hline Altura das plantas (m) & 22 & 0,9 & 0,29 & 22 & 1,0 & 0,23 & 120,0 & 0,004 \\
\hline Número de frutos por planta & 22 & 16,3 & 20,94 & 22 & 13,8 & 13,04 & 26,0 & 0,795 \\
\hline Biomassa dos frutos $(\mathrm{g})$ & 69 & 0,2 & 0,12 & 98 & 0,4 & 0,14 & 1652,0 & $<0,001$ \\
\hline Índice Foliar & 100 & 0,5 & 0,08 & 100 & 0,5 & 0,07 & 5143,0 & 0,727 \\
\hline Largura das folhas (cm) & 100 & 3,6 & 0,77 & 100 & 3,1 & 0,55 & 6863,0 & $<0,001$ \\
\hline Comprimento das folhas $(\mathrm{cm})$ & 100 & 7,6 & 1,57 & 100 & 6,7 & 1,28 & 6774,5 & $<0,001$ \\
\hline Densidade de ocorrência(indivíduos/ m²) & 20 & 1,4 & 1,19 & 20 & 5,0 & 3,66 & 48,5 & $<0,001$ \\
\hline
\end{tabular}

(Fig. 1). A densidade da população de D. alliptica na área de borda de vereda foi significativamente maior que a densidade desta espécie no cerrado $(\mathrm{U}=48,50$; $\mathrm{P}<0,001)$.

Com relação a $B$. intermedia, foram verificadas diferenças fenotípicas entre as áreas para altura dos indivíduos, biomassa dos frutos e índice foliar (Tab. 2). $\mathrm{Na}$ área de cerrado, os valores encontrados na altura dos indivíduos $(\mathrm{U}=340,5 ; \mathrm{P}=0,021)$, biomassa dos frutos $(\mathrm{U}=6090,5 ; \mathrm{P}<0,001)$ e índice foliar $(\mathrm{U}=6171$; $\mathrm{P}=0,004)$ foram maiores em comparação com os valores aferidos na região de borda de vereda. Houve assincronia nos estádios de floração entre as áreas de coleta $\left(\chi^{2}=52,3 ; \mathrm{P}<0,001\right)$, sendo que, também para essa espécie, o ambiente de borda de vereda apresentou uma antecipação nos estádios de floração (Fig. 2). Não foram constatadas diferenças significativas entre as áreas de estudo no número de frutos por planta $(\mathrm{U}=261 ; \mathrm{P}=0,099)$, tamanho das inflorescências $(\mathrm{U}=5364,5 ; \mathrm{P}=0,373)$, largura $(\mathrm{U}=5537 ; \mathrm{P}=0,189)$ e comprimento da folhas $(\mathrm{U}=4769 ; \mathrm{P}=0,572)$. A densidade de ocorrência desta espécie também não diferiu entre as áreas $(\mathrm{U}=246,5 ; \mathrm{P}=0,373)$.

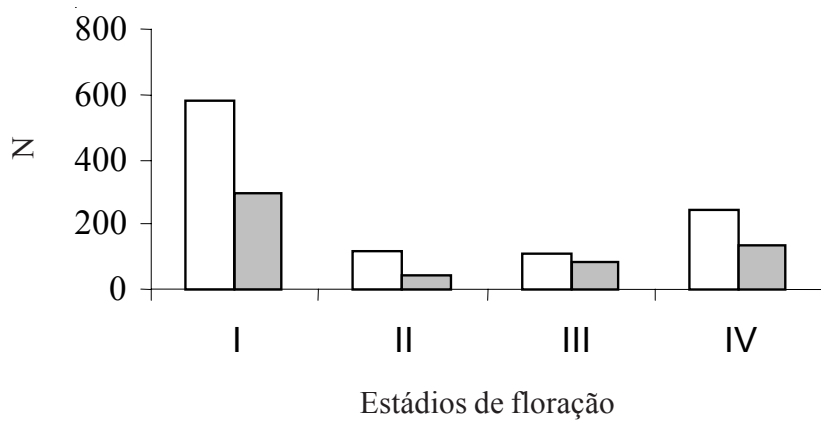

Figura 1. Número de estruturas por estádio de floração de indivíduos de Davilla elliptica A. St.-Hill da Reserva Vegetal do Clube Caça \& Pesca Itororó de Uberlândia, em áreas de cerrado e borda de vereda (I - botões florais, II - botões em pré-antese, III - flores abertas, IV - flores senescentes). $\square$ = borda de vereda, = cerrado.

Tabela 2. Caracterização fenotípica (média e desvio padrão - DP), densidade e comparações (feitas pelo teste de Mann Whitney) de Byrsonima intermedia A. Juss. nas áreas de cerrado sensu stricto e borda de vereda da Reserva Vegetal do Clube Caça \& Pesca Itororó de Uberlândia, MG. (Índice foliar calculado pela razão entre largura e comprimento da folha).

\begin{tabular}{|c|c|c|c|c|c|c|c|c|}
\hline \multirow[t]{2}{*}{ Caracteres analisados } & \multicolumn{3}{|c|}{ Cerrado sensu stricto } & \multicolumn{3}{|c|}{ Borda de Vereda } & \multirow[b]{2}{*}{$\mathrm{U}$} & \multirow[b]{2}{*}{$\mathrm{P}$} \\
\hline & $\mathrm{N}$ & Média & $\overline{\mathrm{DP}}$ & $\mathrm{N}$ & Média & DP & & \\
\hline Altura das plantas (m) & 22 & 1,0 & 0,21 & 22 & 0,9 & 0,16 & 345,5 & 0,021 \\
\hline Número de frutos por planta & 22 & 93,0 & 114,83 & 22 & 44,7 & 43,04 & 261,0 & 0,099 \\
\hline Biomassa dos frutos $(\mathrm{g})$ & 85 & 0,2 & 0,05 & 80 & 0,1 & 0,04 & 6090,5 & $<0,001$ \\
\hline Índice Foliar & 100 & 0,5 & 0,07 & 100 & 0,4 & 0,07 & 6171,0 & 0,004 \\
\hline Largura das folhas $(\mathrm{cm})$ & 100 & 4,7 & 1,02 & 100 & 4,5 & 0,84 & 5537,0 & 0,189 \\
\hline Comprimento das folhas $(\mathrm{cm})$ & 100 & 10,0 & 1,60 & 100 & 10,2 & 1,42 & 4769,0 & 0,572 \\
\hline Comprimento das inflorescências $(\mathrm{cm})$ & 100 & 6,5 & 2,38 & 100 & 6,3 & 3,08 & 5364,5 & 0,373 \\
\hline Densidade de ocorrência(indivíduos $/ \mathrm{m}^{2}$ ) & 20 & 7,3 & 2,40 & 20 & 7,0 & 4,85 & 246,5 & 0,205 \\
\hline
\end{tabular}




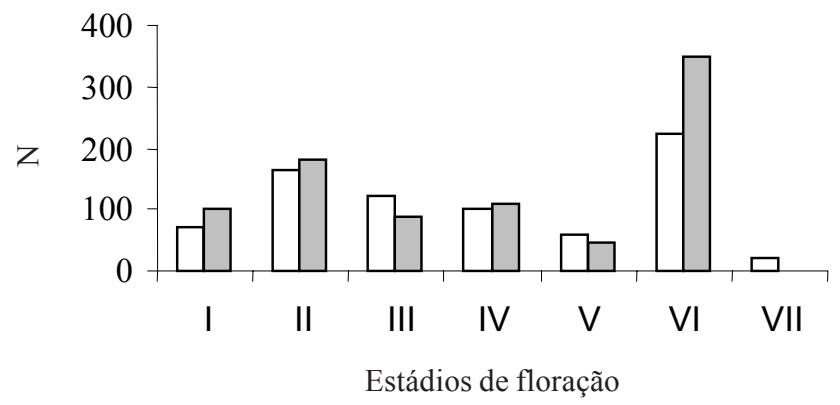

Figura 2. Número de estruturas por estádio de floração de indivíduos de Byrsonima intermedia A. Juss. da Reserva Vegetal do Clube Caça \& Pesca Itororó de Uberlândia, em áreas de cerrado e borda de vereda (I - botões fase 1 (verdes), II - botões fase 2 (verde-amarelados), III - botões fase 3 (amarelos em pré-antese), IV - flores abertas, V - flores senescentes, VI - frutos verdes, VII - frutos maduros). $\square$ = borda de vereda, $\square=$ cerrado.

\section{Discussão}

A heterogeneidade ambiental encontrada na área de transição cerrado-vereda atua na determinação de algumas características morfológicas de D. elliptica. O ambiente de borda de vereda parece ser mais favorável para essa espécie, considerando sua maior densidade de ocorrência, o maior porte (altura) dos indivíduos e os frutos com maior biomassa ali coletados. N.S. Reis (dados não publicados) já havia notado o aspecto raquítico de $D$. elliptica em área de cerradão, o que sinalizava estreita tolerância à baixa luminosidade e conseqüente sucesso em ambientes mais abertos, como é o caso da borda de vereda. Corroborando essa observação, pode ser ainda mencionada a redução nas dimensões do limbo foliar dessa espécie na área de borda de vereda, que apresenta maior exposição à luz solar e menor cobertura vegetal. Assim, o adensamento da vegetação nas áreas ocupadas pelo cerrado sensu stricto poderia estar reduzindo a luminosidade que chega às camadas mais próximas do solo, o que estaria sendo compensado pelo aumento no tamanho das folhas, (Pigliucci et al. 1995; Niinemets 1999; Pons et al. 2001; Hoffmann \& Franco 2003). Ressalta-se, ainda, que nem o número de frutos produzidos por planta nem o índice foliar (que descreve o formato da folha) apresentaram diferenças significativas entre as áreas estudadas, o que sugere maior herdabilidade ou canalização desses caracteres (Stearns 1989).

$B$. intermedia também expressou características morfológicas distintas em resposta à heterogeneidade ambiental. Mas, se por um lado D. elliptica mostrou maior sucesso reprodutivo na área de borda de vereda, $B$. intermedia apresentou maior porte e produziu frutos maiores na área de cerrado sensu stricto.
Comparativamente, variações morfológicas em $B$. intermedia foram observadas em menor número que em D. elliptica. Como não houve diferenças na densidade de ocorrência, pode-se supor que esta seja uma espécie com maior tolerância às variações ambientais entre cerrado e vereda.

Respostas plásticas distintas à heterogeneidade ambiental, como as observadas neste trabalho, já foram descritas inclusive para espécies de mesmo gênero (Sands et al. 2003) e, em certos casos, a expressão substancial de plasticidade em determinados caracteres resultou em confusões na identificação de espécies, que somente puderam ser confirmadas com uso de técnicas de biologia molecular (Sundarasan et al. 2003).

Em comum, D. elliptica e B. intermedia apresentaram padrões assincrônicos de floração, iniciada mais tardiamente nas áreas de cerrado sensu stricto. Berg et al. (1987) e Lima et al. (1989) explicaram padrões similares de floração como resultantes da variação sazonal no nível de umidade do solo. Como as veredas se localizam nas porções mais baixas das vertentes, concentrariam maior teor de umidade ao longo do ano, dada a sua proximidade com o lençol freático e a tendência para o aporte de água pluviométrica por escoamento superficial. Além disto, análises físico-químicas de solo realizadas por Cardoso \& Lomônaco (2003) indicaram maior percentagem de argila nas amostras obtidas na borda da vereda, o que contribuiria para aumento da umidade do solo, considerando a sua grande capacidade de retenção de água. As espécies $C$. canjerana subsp. polytricha e E. calycina, estudadas quanto à sincronia de floração nesta mesma área, também apresentaram etapas do processo de floração adiantadas em áreas de borda de vereda em relação às regiões ocupadas por cerrado sensu stricto (Fuzeto \& Lomônaco 2000; Cardoso \& Lomônaco 2003).

Assim, considerando-se a pequena divergência climática entre as áreas amostradas, visto serem estas adjacentes, padrões edáficos (umidade e percentagem de argila) poderiam ser indicados como os fatores geradores das respostas plásticas observadas.

A literatura especializada tem registrado muitos eventos de variação causados por indução ambiental, como a observada neste trabalho. A rosácea Rubus alceifolius Poir., por exemplo, introduzida em uma ilha do oceano Índico, teve grande sucesso adaptativo em uma grande variedade de hábitats, o que foi atribuído ao seu grande potencial plástico, considerando-se a pequena variabilidade genética do pequeno número de indivíduos fundadores desta população (Baret et al. 
2004). O mesmo estudo apontou que o crescimento vegetativo e a produção de frutos correlacionaram-se com os valores de temperatura dos distintos ambientes ocupados por R. alceifolius. No estudo de Mueckschel \& Otte (2003), a relação entre características morfológicas e fisiológicas de Plantago media L. (Plantaginaceae) e Scabiosa columbaria L. (Droseraceae) e os fatores que determinaram as condições dos ambientes onde as plântulas se desenvolveram foram tão aparentes que diferentes condições ambientais puderam ser monitoradas com acurácia, por meio da análise de seu sucesso de crescimento.

Em áreas de cerrado e mata tropical, Hoffman \& Franco (2003) compararam espécies de plantas nativas que co-ocorrem em ambientes com fitofisionomias variadas e observaram marcantes diferenças nas respostas fenotípicas à intensidade da luz, particularmente no que se refere às dimensões foliares. Embora o componente genético de variabilidade não tenha sido estimado, os resultados apontam para a importância dos fatores ambientais na seleção de caracteres adaptativos em diversas estruturas morfológicas (Hoffman \& Franco 2003).

Os dados obtidos pelo presente estudo confirmam a hipótese de que a heterogeneidade ambiental é uma importante fonte geradora de variações morfológicas de inúmeros caracteres ligados à história de vida das plantas, como salientado por Ungerer et al. (2003) e True et al. (2004). Variações dessa natureza precisam ser consideradas na elaboração de programas de manejo e conservação de áreas protegidas, uma vez que, além de áreas heterogêneas possuírem maior diversidade de nichos, contribuindo para aumento da riqueza de espécies, também promovem aumento da variabilidade fenotípica populacional gerada por indução ambiental. Em termos evolutivos, respostas plásticas à heterogeneidade ambiental representam também modificações adaptativas que alteram padrões da comunidade, expandindo o potencial evolutivo das espécies por meio do aumento da variabilidade fenotípica que, por sua vez, aumenta as possibilidades de sobrevivência e reprodução diante de pressões seletivas (Sultan 2000; Agrawal 2001).

\section{Agradecimentos}

A Thaissa Lopes de Melo e Meyr Pereira Cruz, pelo apoio nas coletas de campo; à Profa. Dra. Ana Angélica Almeida Barbosa, pelo auxílio na identificação das plantas; ao Dr. Heraldo Luís de Vasconcelos, pelas sugestões dadas na elaboração do texto.

\section{Referências bibliográficas}

Agrawal, A.A. 2001. Phenotypic plasticity in the interactions and evolution of species. Science 294: 321-326.

Appolinário, V. \& Schiavini, I. 2002. Levantamento fitossociológico de espécies arbóreas de Cerrado (stricto sensu) em Uberlândia - Minas Gerais. Boletim do Herbário Ezechias Paulo Heringer 10: 57-75.

Baret, S.; Maurice, S.B. \& Strasberg, D.T. 2004. Altitudinal variation in fertility and vegetative growth in the invasive plant Rubus alceifolius Poiret (Rosaceae), on Reunion Island. Plant Ecology 172: 265-273.

Berg, M.U.D.; Lepsch, I.F. \& Sakai, E. 1987. Solos de planícies aluviais do Vale do Ribeira do Iguape, SP. II. Relações entre características físicas e químicas. Revista Brasileira de Ciência do Solo 11: 315-321.

Bradshaw, A.D. 1965. Evolutionary significance of phenotypic plasticity in plants. Pp.115-155. In: E.M. Caspary \& J.M. Thoday (eds.). Advances in Genetics. New York, Academic Press.

Cardoso, G.L. \& Lomônaco, C. 2003. Variações fenotípicas e potencial plástico de Eugenia calycina Camb. (Myrtaceae) em uma área de transição cerrado-vereda. Revista Brasileira de Botânica 26: 131-140.

Castro, J.P.C. 1980. As veredas e a sua proteção jurídica. Análise e Conjuntura 10: 321-331.

Coutinho, L.M. 1978. O conceito do cerrado. Revista Brasileira de Botânica 1: 17-23.

Donohue, K. 2003. Setting the stage: phenotypic plasticity as habitat selection. International Journal of Plant Science 164: 9-92.

Embrapa. 1982. Levantamento de reconhecimento de média intensidade dos solos e avaliação da aptidão agrícola das terras do Triângulo Mineiro. Boletim de Pesquisa 1. Rio de Janeiro, Serviço Nacional de Levantamento e Conservação dos Solos.

Falconer, D.S. 1989. Introduction to Quantitative Genetics. New York, Longman.

Fuzeto, A.P. \& Lomônaco, C. 2000. Potencial plástico de Cabralea canjerana subsp. polytricha (Adr. Juss.) Penn. (Meliaceae) e seu papel na formação de ecótipos em área de cerrado e vereda, Uberlândia, MG. Revista Brasileira de Botânica 23: 169-176.

Hoffmann, W.A. \& Franco, A.C. 2003. Comparative growth analysis of tropical forest and savanna woody plants using phylogenetically independent contrasts. Journal of Ecology 91: 475-484.

Joly, A.B. 1987. Botânica: Introdução à Taxonomia Vegetal. São Paulo, Companhia Editora Nacional.

Lima, S.C.; Rosa, R. \& Feltran Filho, A. 1989. Mapeamento do uso do solo no município de Uberlândia, MG, através de imagens TM/Landsat. Sociedade \& Natureza 1: 127-145.

Lorenzi, H. 2000. Plantas Daninhas do Brasil - Terrestres, Aquáticas, Parasitas e Tóxicas. Nova Odessa, Instituto Plantarum.

Lorenzi, H. \& Matos, F.J.A. 2002. Plantas Medicinais no Brasil: Nativas e Exóticas. Nova Odessa, Instituto Plantarum de Estudos da Flora.

Mares, M.A. 1986. Conservation in South America: Problems, consequences, and solutions. Science 233: 734-739. 
Mückschel, C. \& Otte, A. 2003. Morphological parameters: an approach for the indication of conservation value of low productive calcareous grasslands. Agriculture, Ecosystems and Environment 98: 213-225.

Niinemets, U. 1999. Components of leaf dry mass per area - thickness and density - alter leaf photosynthetic capacity in reverse directions in wood plants. New Phytologist 144: 35-47.

Nimer, E. \& Brandão, A.M.P.M. 1989. Balanço Hídrico e Clima da Região dos Cerrados. Rio de Janeiro, Fundação IBGE.

Panizza, S. 1998. Plantas que Curam (Cheiro de Mato). São Paulo, IBRASA.

Pigliucci, M.; Whitton, J. \& Schlichting, C.D. 1995. Reaction norms of Arabidopisis I. Plasticity of characters and correlations across water, nutrient and light gradients. Journal of Evolutionary Biology 8: 421-438.

Pons, T.L.; Jordi, W. \& Kuiper, D. 2001. Acclimation of plants to light gradients in leaf canopies: evidence for a possible role for cytokinins transported in the transpiration stream. Journal of Experimental Botany 52: 1563-1574.

Pott, A. \& Pott, V.J. 1994. Plantas do Pantanal. Corumbá, Embrapa-CPAP.

Price, T.D.; Qvarnstrom, A. \& Irwin, D.E. 2003. The role of phenotypic plasticity in driving genetic evolution. Proceedings of the Royal Society Biological Sciences series B - 270: 1432-1440.
Sands, R.J.; Brown, N.R. \& Komoulis, A. 2003. Micropropagation of two threatened Tasmanian species of Calociphalus (Asteraceae), with comments on phenotypic plasticity. Australian Journal of Botany 51: 415-420.

Sano, S.M. \& Almeida, S.P. 1998. Cerrado: Ambiente e Flora. Planaltina, Embrapa-CPAC.

Stearns, S.C. 1989. The evolutionary significance of phenotypic plasticity. Bioscience 39: 436-445.

Sultan, S.E. 2000. Phenotypic plasticity for plant development, function and life history. Trends in Plant Science 5: 537-542.

Sundarasan, V.; Rajkumar, S.; Shasany, A.K.; Darokar, M.P. \& Khanuja, S.P.S. 2003. Revisiting taxonomic gaps in some medicinal plant species: Molecular aids to resolve phylogenetic status. Journal of Medicinal and Aromatic Plant Sciences 25: 134-148.

Thompson, J.D. 1991. Phenotypic plasticity as a component of evolutionary chance. Trends in Ecology and Evolution 6: 246-249.

True, H.L.; Berlin, I. \& Lindquist, S.L. 2004. Epigenetic regulation of translation reveals hidden genetic variance to produce complex traits. Nature 431: 184-187.

Ungerer, M.C.; Halldorsdottir, S.S. \& Purugganan, M.D. 2003. Genotype-environment in Arabidopsis thaliana. Genetics 165: 353-365.

Zar, J.H. 1984. Biostatistical Analysis. Upper Saddle River, Prentice-Hall. 